\title{
COHERENT EFFECT IN THE PREFERENTIAL ACCELERATION OF RELATIVISTIC SOLAR HEAVY COSMIC RAY NUCLEI
}

\author{
SHAHINAZ M. A. YOUSEF \\ Astronomy Dept., Cairo University, Cairo, Egypt
}

\begin{abstract}
Solar Phys.). The observed enrichment of heavy nuclei in the composition of relativistic cosmic rays including solar, may be explained on the basis of a coherent accelerating mechanism. This mechanism requires the existence of high intensity relativistic electron beams or preferably blobs (with number density $\geqslant 10^{11}$ electrons $\mathrm{cm}^{-3}$ ) in the accelerating solar regions. The radius of each blob should be less than its plasma wavelength, i.e. of the order of $\mathrm{cm}$.

If such electron blobs do exist, then each would be partially self focused by its magnetic attraction force due to the pinch effect which counteract $(V / c)^{2}$ of the coulomb repulsive force of the electrons. As these blobs propagate through the solar atmosphere they will electrostatically pick up a suitable number of positive ions, strip them of their electrons and capture them in their deep potential wells. This seeding of these electron blobs by positive ions would render them stable at least over short periods.

These captured positive ions would have no choice but to travel with the blobs with their relativistic velocities. Assuming the energy of each electron in the blob to be $1 \mathrm{MeV}$, then the corresponding energy of a captured proton would be $1836 \mathrm{MeV}$ and that of say an iron nucleus would be $102.8 \mathrm{GeV}$.

These positive ions are actually accelerated by the internal coherent electric field of the blob's electrons. What ever happens to each blob after the acceleration of the positive ions should not in principle affect these ions.

Since the capture of positive ions is electrostatic in nature, the blobs preferentially collects high $Z$ nuclei.

Briefly these electron blobs can serve as vehicles for the preferential acceleration of heavy cosmic ray nuclei.

The principle of this mechanism has recently been already applied in a modified way in the electron ring accelerators and its possibility, for the acceleration of cosmic rays has been briefly pointed out in 1956 by Veksler and by Budker, independently.
\end{abstract}

\section{DISCUSSION}

Meyer: The relativistic electron cloud needs only relatively few ions for charge compensation. Now usually in the solar plasma there are many ions available, which is different from the situation in the plasma accelerators. One wonders whether under such circumstances the ions really have to travel along with the relativistic electron bunch. I could imagine that the ions would basically just stay where they are and are only effected by the passing electrons through a kind of polarization field, so that no ions would travel along but only a kind of polarization wave pattern. Did you investigate this situation?

Yousef: I should expect that there must be a threshold or some critical condition that allows the electron cloud to suck only a smaller number of the surrounding ions, preferably the heavy ions, strip them 
of their electrons and trap them inside the cloud or drag some ions that settle near its tail. The very short time of acceleration (which I guess is less than $10^{-5} \mathrm{~s}$ limits the number of trapped ions to a small percentage of the total electron number forming the cloud. I would like to stress here that the electron cloud does not have to necessarily be stable. However, I did not investigate the polarization field you mentioned.

Smith: There are two problems which I can see with your model. (1) How do you generate beams with densities greater than $10^{11} \mathrm{~cm}^{-3}$ in a corona where acceleration occurs in regions where the density is less than $10^{10} \mathrm{~cm}^{-3}$ ? Your beams, especially the ones with velocities of $0.5 c$ should be very unstable. Do you have any idea how to stabilize them.

Yousef: In pinching, the plasma attains higher densities.

Smith: I am familiar with the work on relativistic electron beams in the laboratory. What I am really worried about is the generation of such beams under solar conditions. All laboratory pinches with which I am familiar which lead to particle acceleration inevitably involve a current sheet and these don't give very coherent dense pulses of particles.

Krall (to Smith): There is some precedent in laboratory plasmas for propagating electron beams a distance of very many instability growth lengths. One mechanism would be to convert a small fraction of the beam energy into temperatures perpendicular to the beam. This increases the Debye length and can lead to finite size effects which stabilize the non-neutralized beam.

Laboratory experiments have shown some preferential acceleration of a small number of ions in pinch experiments. I agree that the conditions would have to be pretty special to produce this.

Another coherent acceleration mechanism is inverse Cerenkov radiation. In the rest frame of the electron beam the ions move super-fast, emit plasma waves and slow down by recoil. In the lab frame this corresponds to ion acceleration. 